\title{
Alpha-ketoglutarate affects murine embryo development through metabolic and epigenetic modulations
}

\author{
Zhenzhen Zhang ${ }^{1, *}$, Changjiu He ${ }^{1,2, *}$, Lu Zhang ${ }^{1}$, Tianqi Zhu¹, Dongying Lv ${ }^{1}$, Guangdong Li ${ }^{1}$, \\ Yukun Song ${ }^{1,3}$, Jing Wang ${ }^{1}$, Hao Wu ${ }^{1}$, Pengyun Ji ${ }^{1}$ and Guoshi Liu ${ }^{1}$ \\ ${ }^{1}$ National Engineering Laboratory for Animal Breeding, Key Laboratory of Animal Genetics and Breeding of the \\ Ministry of Agriculture, Beijing Key Laboratory for Animal Genetic Improvement, College of Animal Science and \\ Technology, China Agricultural University, Beijing, China, ${ }^{2}$ Key Laboratory of Agricultural Animal Genetics, Breeding \\ and Reproduction, Education Ministry of China, College of Animal Science and Technology, Huazhong Agricultural \\ University, Wuhan, China and ${ }^{3}$ College of Animal Science and Technology, Xinjiang Agricultural University, \\ Wulumuqi, China
}

Correspondence should be addressed to G Liu; Email: gshliu@cau.edu.cn

*(Z Zhang and C He contributed equally to this work)

\begin{abstract}
$\alpha$-Ketoglutarate $(\alpha-K G)$ is an intermediary metabolite in the tricarboxylic acid (TCA) cycle and functions to inhibit ATPase and maintain the pluripotency of embryonic stem cells (ESCs); however, little is known regarding the effects of $\alpha$-KG on the development of preimplantation embryos. Herein, we report that $\alpha-\mathrm{KG}(150 \mu \mathrm{M})$ treatment significantly promoted the blastocyst rate, the number of inner cell mass (ICM) cells and foetal growth after embryo transfer. Mechanistic studies revealed two important pathways involved in the $\alpha-K G$ effects on embryo development. First, $\alpha-K G$ modulates mitochondria function by inducing relatively low ATP production without modification of mitochondrial copy number. The relatively low energy metabolism preserves the pluripotency and competence of the ICM. Second, $\alpha-K G$ modifies epigenetics in embryos cultured in vitro by affecting the activity of the DNA demethylation enzyme TET and the DNA methylation gene Dnmt3a to increase the ratio of $5 \mathrm{hmC} / 5 \mathrm{mC}$ ratio. Elevation of the $5 \mathrm{hmC} / 5 \mathrm{mC}$ ratio not only promotes the pluripotency of the ICM but also leads to a methylation level in an in vitro embryo close to that in an in vivo embryo. All these functions of $\alpha-K G$ collectively contribute to an increase in the number of ICM cells, leading to greater adaptation of cultured embryos to in vitro conditions and promoting foetal growth after embryo transfer. Our findings provide basic knowledge regarding the mechanisms by which $\alpha-K G$ affects embryo development and cell differentiation.

Reproduction (2019) 158 123-133
\end{abstract}

\section{Introduction}

$\alpha$-Ketoglutarate $(\alpha-K G)$, a central carbon metabolite in the TCA cycle, is produced from isocitrate by isocitrate dehydrogenase (IDH). Recently, Chin et al. first discovered that $\alpha-K G$ could delay ageing and increase the lifespan of adult $C$. elegans by inhibiting ATP5B, the beta subunit of the catalytic core of ATP synthase, and the TOR pathway, thereby mimicking the effect of dietary restriction on longevity (Chin et al. 2014). In addition to being a metabolite in the TCA cycle, $\alpha-K G$ is a substrate for a large family of dioxygenases, including JmjC-domain-containing histone demethylases (JHDMs) and ten-eleven translocation (TET) enzymes (Carey et al. 2015). TET proteins are the key epigenetic modification enzymes for activation of DNA demethylation (Tahiliani et al. 2009). The function of these Fe(II)- and $\alpha$-KGdependent enzymes is to remove 5-methylated cytosine (5mC) by converting it to 5-hydroxymethylcytosine
(5hmC) and other oxidised derivatives (Ficz et al. 2011, Ito et al. 2011, Jurkowski et al. 2011). TET proteins regulate locus-specific demethylation in embryonic stem cells (ESCs) (Ficz et al. 2013, von Meyenn et al. 2016), and their depletion reduces the expression of pluripotency genes and increases methylation levels at their promoters (Ficz et al. 2011, Williams et al. 2011), ultimately leading to decreased cell pluripotency and cell differentiation. Furthermore, overexpression of TET1 and TET2 dramatically enhances induced pluripotent stem cell (iPSC) reprogramming in a catalytically dependent manner (Chen et al. 2013). Interestingly, $\alpha$ KG can maintain cell pluripotency by regulating the extent of histone K3K27me3 and TET-dependent DNA demethylation (Carey et al. 2015).

Mouse embryo preimplantation development is a widely used mammalian model to study cell differentiation and cell fate decisions. The two earliest cell fate decisions in the preimplantation embryo are 
(1) formation of the trophectoderm (TE) and the inner cell mass (ICM) during early development from the morula to the blastocyst and (2) subsequent formation of the primitive endoderm (PE) and the epiblast (EPI) as the blastocyst prepares for implantation (ZernickaGoetz et al. 2009). The ICM expresses OCT4, SOX2 and NANOG transcription factors, which resist differentiation and maintain pluripotency. The ICM will eventually give rise to the definitive structures of the foetus. TE cells, which develop into the placenta, are differentiated cells with upregulated expression of the transcription factors CDX2 and EOMES. Along with the cell fate decision of early embryonic cells, dramatic changes in methylation occur in these three cell types (Smith et al. 2014, Legault et al. 2018). Several researchers have demonstrated that TET1 is required for ICM cell specification in blastocysts (Ito et al. 2010, Williams et al. 2011). In view of $\alpha$-KG regulation of TET proteins and the level of methylation of whole genome DNA in stem cells (Carey et al. 2015), we speculate that $\alpha-K G$ may play an important role in early embryonic development and cell fate decisions.

The major objective of this study was to determine the effects of $\alpha-K G$ on the developmental potential of early embryos. To investigate the mechanisms, the functional status of mitochondria was examined. Furthermore, RNAseq was conducted, and the TET enzyme activity in each group was determined. The results indicate that supplementation of $\alpha-K G$ in the in vitro embryo culture system is beneficial for early embryo development.

\section{Materials and methods}

The study strictly followed the protocol of the Animal Welfare Committee of China Agricultural University (permission number: CAU20150915-1). Pregnant mare serum gonadotropin (PMSG) and human chorionic gonadotropin (hCG) were purchased from Ningbo Hormone Products Co., Ltd. (Zhejiang, China). Alpha-ketoglutarate and other reagents, unless specified, were purchased from Sigma-Aldrich Chemical Co.

\section{Embryo collection}

ICR mice (7-8 weeks old) were purchased from Beijing Vital River Laboratory Animal Technology Co., Ltd. and raised in a room with a controlled temperature $\left(20-22^{\circ} \mathrm{C}\right)$ and a 12:12 h light/dark cycle (lights on at 08:00 h). After 1 week of acclimation, superovulation was induced in female mice via injection of 7.5 IU PMSG (i.p.) followed by 7.5 IU hCG (i.p.) $48 \mathrm{~h}$ later. Immediately, the female mice were allowed to mate. The next day, females with bolts were killed in the afternoon by cervical dislocation. The pronuclear embryos were collected from the oviducts with M2 medium, digested with $0.1 \%$ hyaluronidase and washed three times with the M2 medium. Embryo handling was performed in the laboratory at ambient temperature $\left(25 \pm 0.5^{\circ} \mathrm{C}\right)$. Media and embryos were maintained at $37^{\circ} \mathrm{C}$ on a warming plate (Wenesco, Inc., Chicago, IL, USA) over the course of the operation. The pronuclear embryos were evaluated under a stereomicroscope, and normal embryos were selected for further study.

\section{Pronuclear culture}

Pronuclear embryos were cultured in KSOM medium (20-30 embryos/drop $/ 60 \mu \mathrm{L}$ ) containing different concentrations of $\alpha$ KG $(0,100,150,200$ and $300 \mu \mathrm{M})$ in an incubator $\left(5 \% \mathrm{CO}_{2}\right.$, $37^{\circ} \mathrm{C}$ and $100 \%$ humidity). The two-cell embryo rate at $24 \mathrm{~h}$ and the blastocyst rate at 108-112 h after culture initiation were recorded. The two-cell, four-cell, morula and blastocyst stage embryos were collected for subsequent experiments.

\section{Detection of ROS in embryos}

Two-cell, four-cell, morula and blastocyst embryos were used to detect intracellular reactive oxygen species (ROS) levels. Briefly, 2', $7^{\prime}$-dichlorohydrofluorescein diacetate (DCHFDA) (Beyotime Institute of Biotechnology, Jiangsu, China) was used as a green fluorescence indicator. Ten to fifteen embryos per group were incubated in $10 \mu \mathrm{M}$ DCHFDA diluted with M2 medium in the dark for $30 \mathrm{~min}$ and then were washed with PBS with $0.1 \%$ PVA three times. The green fluorescence was measured using an epifluorescence microscope (TE300, Nikon) with UV filters ( $460 \mathrm{~nm}$ for ROS), and images were saved as graphic files in TIF format. The fluorescence intensities of the embryos was analysed using ImageJ software.

\section{Apoptotic (TUNEL) assay and total cell count}

In a TUNEL assay, when genomic DNA is cleaved, a fluorescein (FITC)-labelled dUTP molecule (fluoresceindUTP) is transferred to the exposed $3^{\prime}-\mathrm{OH}$, and the transfer is catalysed by terminal deoxynucleotidyl transferase (TdT). The fluorescence can then be observed by microscopy or flow cytometry, and thus, apoptotic cells can be identified. Briefly, blastocysts were washed three times in PBS with $0.1 \%$ PVA and were fixed in $4 \%$ paraformaldehyde solution overnight at $4^{\circ} \mathrm{C}$. Embryo membranes were permeabilised in $0.5 \%$ TritonX$100-$ PBS- $0.1 \%$ PVA for $1 \mathrm{~h}$ at room temperature. Then, fixed embryos were incubated in TUNEL reaction medium (In Situ Cell Death Detection Kit, Fluorescein; Roche) for $1 \mathrm{~h}$ at $37.5^{\circ} \mathrm{C}$ in the dark. After the reaction was stopped, the embryos were washed in PBS-0.1\% PVA three times and mounted on glass slides with DAPI (CA 94010, Vector laboratories, Inc. Burlingame, USA) for total cell counts. Whole-mount embryos were examined under a fluorescence microscope (FV1000, Olympus) with TUNEL and DAPI staining. The apoptotic rate was calculated as follows: apoptotic rate $=$ (number of TUNELpositive nuclei/total number of nuclei) $\times 100 \%$.

\section{Blastocyst transfer}

Based on a previously reported embryo transfer method (Wang et al. 2013), female mice were mated with vasectomised males, and the females with vaginal plugs were selected as recipient mice. On day 2.5 after formation of the vaginal plugs, the recipient mice were anaesthetised with sodium pentobarbital via intraperitoneal injection. Twelve blastocysts 
from pronuclear embryos cultured with or without $150 \mu \mathrm{M}$ $\alpha-K G$ were transplanted into the recipient mice. Once the embryo transplantation was completed, the mice were raised in a cage with clean autoclaved sawdust to avoid any stress or noise that may lead to abortion or even cannibalism of the pups. The pups were born approximately 16 days after implantation. The litter size, birth weight and weaning weight were recorded and statistically analysed.

\section{RNA extraction and quantitative real-time PCR}

Total RNA was extracted from the blastocysts with TRIzol (Invitrogen Inc). The mRNA levels of Sirt1, Caspase 3, Oct4, Sox2, Nanog, Dnmt3a, Dnmt3b, Tet1 and Tet2 were measured by qRT-PCR using a One Step SYBR PrimeScript RT-PCR Kit (TaKaRa Bio. Inc.) and a LightCycler system (Roche Applied Science). The relative expression levels of the target genes were normalised to the expression level of GAPDH in each sample using the $2^{-\Delta \Delta C t}$ method. The primer pairs of analysed mRNA are described in Supplementary Table 1 (see section on supplementary data given at the end of this article).

\section{Immunofluorescence staining and ICM/TE cell number analysis}

Blastocysts were fixed in 4\% paraformaldehyde, permeabilised using $0.5 \%$ Triton-100 in PBS, blocked in 1\% BSA-PBS- $0.1 \%$ PVA for $1 \mathrm{~h}$ at room temperature and incubated with antiOCT4 antibody (1:400; Santa Cruz Biotechnology) and anti-CDX2 antibody (1:200; BioGenes, Berlin, Germany) overnight at $4^{\circ} \mathrm{C}$. DNA was denatured with $4 \mathrm{M} \mathrm{HCl}$ for $10 \mathrm{~min}$, neutralised with $\mathrm{pH} 8.0$ Tris- $\mathrm{HCl}$ for $15 \mathrm{~min}$, blocked in 1\% BSA-PBS-0.1\% PVA overnight and incubated with anti-5mC antibody (1:200; Abcam) and anti-5hmC antibody (1:500; Abcam) for $1 \mathrm{~h}$ at room temperature. After washing with PBS-0.1\% PVA, the reaction was continued using Alexa Fluor 594 Goat Anti-Rabbit IgG Antibody (anti-rabbit; Invitrogen) and Alexa Fluor 488 Goat Anti-Mouse IgG Antibody (anti-mouse; Invitrogen) at a 1:1000 dilution for $1 \mathrm{~h}$ at room temperature. After the reaction was stopped, the embryos were washed in PBS-0.1\% PVA and mounted on glass slides with mounting medium for fluorescence staining with DAPI (CA 94010, Vector Laboratories, Inc.) followed by total cell counts. Embryos were imaged immediately on an epifluorescence microscope. The number of ICM and TE cells was determined according to the number of cells positive for immunofluorescence-labelled OCT4 and CDX2, respectively. The fluorescence intensity of $5 \mathrm{mC}$ and $5 \mathrm{hmC}$ labelling was analysed using ImageJ software.

\section{Mitochondrial membrane potential assay}

The mitochondrial membrane potential (MMP) in the blastocysts was measured using JC-1 (Beyotime Institute of Biotechnology). JC-1 has two fluorescence emission peaks (red fluorescence indicating high MMP and green indicating low MMP). Briefly, blastocysts in each group were exposed to $10 \mu \mathrm{g} / \mathrm{mL} \mathrm{JC}-1$ at $37^{\circ} \mathrm{C}$ for $15 \mathrm{~min}$ in the dark and then washed with PBS-0.1\% PVA three times. The distribution of JC-1 dimers (red fluorescence) and monomers (green fluorescence) was detected using an epifluorescence microscope. The fluorescence intensity was analysed using ImageJ software, and the ratio of red to green fluorescence was used to determine MMP.

\section{ADP/ATP level assay}

Blastocysts were washed with PBS-0.1\% PVA, and seven morulas/group were collected for ADP/ATP ratio measurement. The ATP and ADP levels were determined using an ADP/ ATP ratio assay kit (MAK135, Sigma-Aldrich) according to the manufacturer's instructions. Briefly, the blastocysts were transferred into wells of a 96-well plate along with $45 \mu \mathrm{L}$ ATP reagent buffer, $1 \mu \mathrm{L}$ substrate, $1 \mu \mathrm{L}$ cosubstrate and $1 \mu \mathrm{L}$ ATP enzyme and placed at room temperature for $1 \mathrm{~min}$. Then, a luminometer was used to detect the $\mathrm{RLU}_{\mathrm{A}}$ value for the ATP assay. The plate was incubated for an additional $10 \mathrm{~min}$. After the 10-min incubation, luminescence was recorded for ATP $\left(R L U_{B}\right)$ assessment. This measurement provides the background prior to measuring ADP (i.e., the residual ATP signal). Immediately following the $\mathrm{RLU}_{\mathrm{B}}$ reading, $5 \mu \mathrm{L}$ ADP reagent ( $1 \mu \mathrm{L}$ ADP enzyme and $5 \mu \mathrm{L}$ water) was added to each well and mixed by tapping of the plate or pipetting. After $1 \mathrm{~min}$, the luminescence $\left(R L U_{C}\right)$ was read. The ADP/ATP ratio was calculated using the following formula: ADP/ATP ratio $=\left(R L U_{C}-R L U_{B}\right) / R L U_{A}$.

\section{Mitochondria copy number assay}

Based on a previous study (Ren et al. 2015), the nDNA and mtDNA copy numbers were determined by assessing the level of the nuclear-encoded $\beta$-actin gene ( $\beta$-actin) and the mitochondria-encoded NADH dehydrogenase subunit 5 gene (MT-ND5), respectively. Both the $\beta$-actin gene and the MT-ND5 gene were amplified and cloned into a pEASYTM-T5 Zero cloning vector (TransGen Biotech). Standard curves were determined using ten-fold serial dilution recombinant plasmids. The overall number of mtDNA copies per blastocyst was calculated as follows: $m$ tDNA copies per blastocyst $=M T$ ND5 copy number/( $\beta$-actin copy number/2).

\section{TET hydroxylase activity quantification}

The blastocysts were washed with PBS-0.1\% PVA three times and broken by freeze-thawing with liquid nitrogen to release the total protein. The total protein was divided into two parts; one part was used to detect the concentration of protein extract with a BCA assay, and the rest was used to detect the activity of TET using a TET hydroxylase activity quantification kit (ab156913, Abcam) according to the manufacturer's instruction. The protein extract was incubated with substrate in assay buffer. The wells were washed, and then, the capture antibody was added. The wells were washed again, and then, the detection antibody and enhancer solution were added. A fluoro-development solution was added for fluorescence development and to measure the RFU. 


\section{Total protein measurement - BCA protein assay}

Protein amounts were measured using a BCA Protein Assay Kit (P0010, Beyotime Biotechnology) according to the manufacturer's instructions (Wu et al. 2004). Both standard dilution series and samples were prepared in duplicate and incubated for $1 \mathrm{~h}$ at $37^{\circ} \mathrm{C}$. Optical density was measured at $562 \mathrm{~nm}$ using a Synergy HTX multimode reader (BioTek, Swindon, UK).

\section{High-throughput RNA sequencing and biological analysis}

Fresh blastocysts from different groups were stored in $6 \mu \mathrm{L}$ of SMART-Seq ${ }^{\text {TM }}$ 4 Kit pyrolysis fluid (after volume measurement using a SMART-Seq ${ }^{\text {TM }}$ v4 Ultra ${ }^{\text {TM }}$ Low Input RNA Kit) for cell lysis and synthesis of first-strand cDNA for sequencing (the total RNA samples were stored in RNase-free water directly for synthesis of first-strand cDNA). Then, the first-strand cDNA was amplified via full-length LD-PCR, and the doublechain cDNA (ds cDNA) was purified with AMPure XP beads and quantified using Qubit. The ds cDNA was interrupted via ultrasound using a Covaris system. The interrupted twostranded short fragments were repaired at the end, and the poly-A tail and joint were added to the sequencing connector. Then, AMPure XP beads were used for purification. Fragments ( 200 bp) were selected and enriched by PCR for generation of a cDNA library. After the library was constructed, first, Qubit2.0 was used for initial quantification, and the library was diluted to $2 \mathrm{ng} / \mu \mathrm{L}$; then, AGILENT2100 was used to detect the library insert size. When the insert size was consistent with the expected size, the effective concentration of the library was accurately quantified using the Q-PCR method (effective concentration of library $>2 \mathrm{nM}$ ) to ensure the quality of the library. After inspection of the library, according to the requirements of effective concentration and target data volume, different libraries were utilised for HiSeq sequencing. The sequenced reads were mapped to the reference mouse genome (mm10). The gene expression intensity was normalised as fragments per kilo-base of exon per million fragments mapped (FPKM). Gene ontology (GO; http://www. geneontology.org) was used to annotate biological themes, and the Kyoto Encyclopedia of Genes and Genomes (KEGG; http://www.genome.jp/kegg/) was used to find the associated pathways. Novogene (Beijing, China) helped complete the sequencing and the data annotation.

The input data of the differentially expressed gene analysis were the readcount data that were obtained from the gene expression level analysis. The analysis was mainly divided into three parts: (1) normalisation of the readcount; (2) calculation of the hypothesis test probability ( $P$ value) according to the model (DESeq) and (3) performance of a multiple hypothesis test calibration to obtain the FDR value (error discovery rate). DESeq software was used to analyse the differential expression of genes in different situations. To increase the power to detect biologically meaningful functions, a relative relaxed criterion of a two-fold change and a $P$ value $<0.05$ were used to filter differentially expressed genes for biological analysis.

\section{Statistical analysis}

The data are presented as the means \pm S.E.M. One-way analysis of variance followed by Tukey's method and a chi-square test were applied to analyse the data using SPSS 20.0 statistical software (SPSS Inc.). Statistical significance was set at $P<0.05$.

\section{Results}

\section{Effects of different $\alpha-K G$ concentrations on in vitro development of murine pronuclear embryos}

To investigate the potential effects of $\alpha$-KG on in vitro development of murine pronuclear embryos, a total of 1326 pronuclear embryos were used. The results showed that the cleavage rate of the pronuclear embryos treated with $\alpha-K G$ was not significantly different from that of the control embryos (Fig. 1A). The blastocyst rates of pronuclear embryos treated with $\alpha-\mathrm{KG}$ at 100, 150,


C
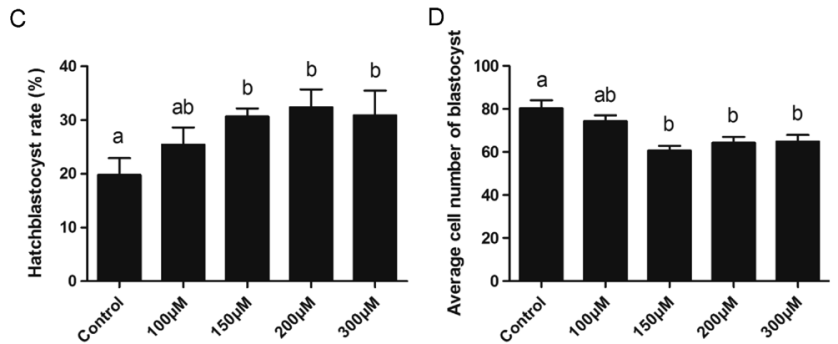

$\mathrm{E}$

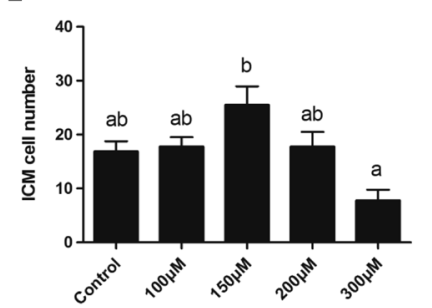

Figure 1 Effects of different $\alpha-K G$ concentrations on in vitro development of murine pronuclear embryos. (A) Cleavage rates (control, $n=302 ; 100 \mu \mathrm{M}, n=302 ; 150 \mu \mathrm{M}, n=285 ; 200 \mu \mathrm{M}, n=256$; $300 \mu \mathrm{M}, n=181$ ); (B) blastocyst rates (control, $n=302 ; 100 \mu \mathrm{M}$, $n=302 ; 150 \mu \mathrm{M}, n=285 ; 200 \mu \mathrm{M}, n=256 ; 300 \mu \mathrm{M}, n=181) ;(\mathrm{C})$ hatch-blastocyst rates (control, $n=302 ; 100 \mu \mathrm{M}, n=302 ; 150 \mu \mathrm{M}$, $n=285 ; 200 \mu \mathrm{M}, n=256 ; 300 \mu \mathrm{M}, n=181)$; (D) total blastocyst cell number (control, $n=35 ; 100 \mu \mathrm{M}, n=48 ; 150 \mu \mathrm{M}, n=51 ; 200 \mu \mathrm{M}$, $n=65 ; 300 \mu \mathrm{M}, n=27)$; (E) inner cell number (control, $n=23$; $100 \mu \mathrm{M}, n=9 ; 150 \mu \mathrm{M}, n=6 ; 200 \mu \mathrm{M}, n=19 ; 300 \mu \mathrm{M}, n=17)$. Different letters represent statistically significant differences $(P<0.05) . n=$ number of embryos. 
200 and $300 \mu \mathrm{M}$ were $62.70 \pm 5.74 \%, 68.67 \pm 2.43 \%$, $62.50 \pm 5.24 \%$ and $61.33 \pm 8.46 \%$, respectively; the value of the group with $150 \mu \mathrm{M} \alpha-\mathrm{KG}$ treatment was significantly higher than that of the control group $(54.40 \pm 5.03 \%, P<0.05)$ (Fig. 1B). The hatch-blastocyst rates of the $\alpha-K G(150,200$ and $300 \mu \mathrm{M})$ groups were significantly higher than those of the control group (Fig. 1C) $(P<0.05)$. Surprisingly, the average blastocyst cell number in these $\alpha-K G$ groups $(150,200$ and $300 \mu \mathrm{M})$ was significantly decreased compared with that of the control (Fig. 1D) $(P<0.05)$. The ICM in the $150 \mu \mathrm{M} \alpha-\mathrm{KG}$ group was remarkably higher than that in the control group (Fig. 1E) $(P<0.05)$.

\section{$\alpha-K G$ promotes birth and weaning weights after embryo transfer}

To examine whether the quality of the $\alpha$-KG-treated embryos was better than that of control embryos, an embryo transfer method was used. The early blastocysts, which were cultured with or without $\alpha-K G(150 \mu \mathrm{M})$ for 3.5 days, were transferred into recipient mice. The birth and weaning weights in the $\alpha-K G(150 \mu \mathrm{M})$ group were $1.941 \pm 0.053 \mathrm{~g}$ and $12.650 \pm 0.538 \mathrm{~g}$, respectively, which were significantly higher than those in the control group $(1.783 \pm 0.054 \mathrm{~g}, \quad 9.548 \pm 0.184 \mathrm{~g} ; \quad P<0.05)$ (Fig. $2 \mathrm{~B}$ and $\mathrm{C}$ ). The litter size in the $\alpha-K G$ group $(6.143 \pm 0.799)$ was larger than that in the control group $(5.625 \pm 0.998)$; however, this difference failed to reach statistical significance (Fig. 2A) $(p>0.05)$.
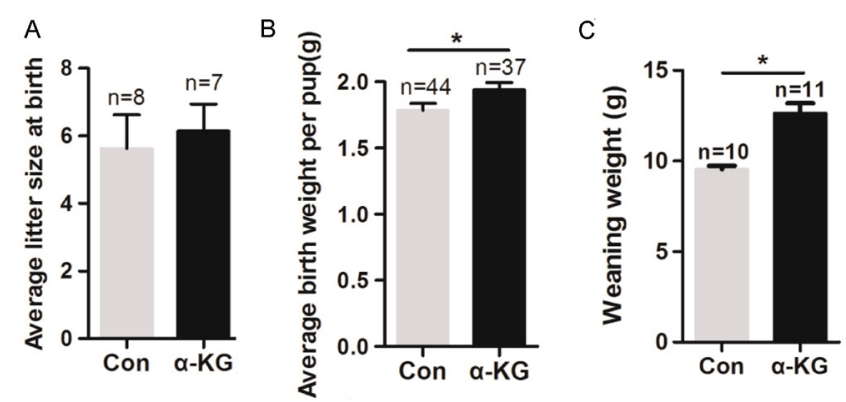

D

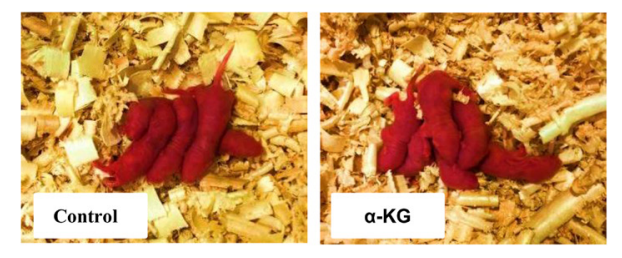

Figure 2 Effects of $\alpha$-KG on embryo transfer after in vitro culture for 3.5 days. (A) Average litter size at birth, $n=$ number of litters; (B) average birth weight per pup, $n=$ number of pups; (C) average weaning weight per pup, $n=$ number of pups; (D) images of newborn mice from transplanted embryos in the control and $\alpha-K G$ groups $*(P<0.05)$.

\section{$\alpha-K G$ increases the cell number and percent of inner cell mass}

The cell number and percent of ICM in the group treated with $\alpha-K G(150 \mu \mathrm{M})$ were $21.85 \pm 0.82$ and $34.87 \pm 1.35 \%$, respectively, which is significantly higher than those in the control group $(19.10 \pm 1.40$ and $26.16 \pm 1.86 \%$, respectively) $(P<0.05)$ (Fig. $3 \mathrm{~B}$ and $\mathrm{C}$ ). The small difference in ICM cells shown in Figs $1 \mathrm{E}$ and 3B may be due to different experimental repetitions. The TE number and percentage in the $\alpha-K G$ group were $43.56 \pm 1.75$ and $65.13 \pm 1.35 \%$, respectively, which are significantly lower than those in the control group $(54.95 \pm 2.18$ and $74.00 \pm 1.82 \%$, respectively) $(P<0.05)$ (Fig. 3D and E). Given that ICM cells are deeply associated with naïve pluripotency and the establishment of ESCs, we detected the expression levels of pluripotency-related genes (Oct4, Sox2 and Nanog) in the control and $\alpha$-KG-treated embryos using qRT-PCR. The results indicated that the relative expression levels of the Sox2 and Nanog genes showed no significant changes, while Oct4 and Sirt1 expression levels in the $\alpha-K G$-treated group were significantly elevated (Fig. 3F).

\section{$\alpha$-KG decreases the ATP level without changing the mitochondrial copy number in embryos}

Based on a previous paper (Chin et al. 2014), we speculated that $\alpha-K G$ might inhibit mitochondrial function to improve the quality of the ICM. The most direct indicators of mitochondria function, including ATP production, MMP and mitochondria copy number, were investigated. The results showed that $\alpha-K G$ treatment significantly decreased the RLU of ATP in embryos compared with that in the controls $(162.4 \pm 5.51$ vs $116.7 \pm 8.28)(P<0.05)$ (Fig. 4 C), while no significant changes were found in the RLU of ADP $(48.75 \pm 7.09$ control vs $36.73 \pm 5.14 ; P>0.05)$ or in the ADP/ ATP ratio $(30.43 \pm 4.45 \%$ control vs $33.37 \pm 5.08 \%$; $P>0.05)$ between the two groups (Fig. $4 \mathrm{D}$ and E). The mitochondrial copy number between the control and $\alpha$ KG group was not remarkably different $(487.7 \pm 137.3$ control vs $531.9 \pm 116.2 ; P>0.05$ ) (Fig. $4 \mathrm{~F}$ ). $\alpha-K G$ significantly increased the MMP compared with that in the control group $(3.93 \pm 0.60$ vs $1.34 \pm 0.20 ; P<0.05)$ (Fig. 4A and $\mathrm{B}$ ). In addition, the relative fluorescence intensity values of DCFHDA in the two-cell, four-cell and morula stage embryos in the $\alpha-K G$ groups were $0.025 \pm 0.0014,0.082 \pm 0.0033$ and $0.086 \pm 0.0084$, respectively, which were significantly higher than the values in the corresponding control groups $(0.021 \pm 0.0010,0.062 \pm 0.0064$ and $0.051 \pm 0.0069$, respectively; $P<0.05$ ) (Supplementary Fig. $2 \mathrm{C}$, D and $E)$. The phenomenon was reversed in the blastocyst stage $(0.039 \pm 0.0016$ vs $0.031 \pm 0.0024 ; \quad P<0.05)$ (Supplementary Fig. 2F). Both the TUNEL assay and Caspase-3 expression showed no significant difference 
A



B



C



D
DAPI
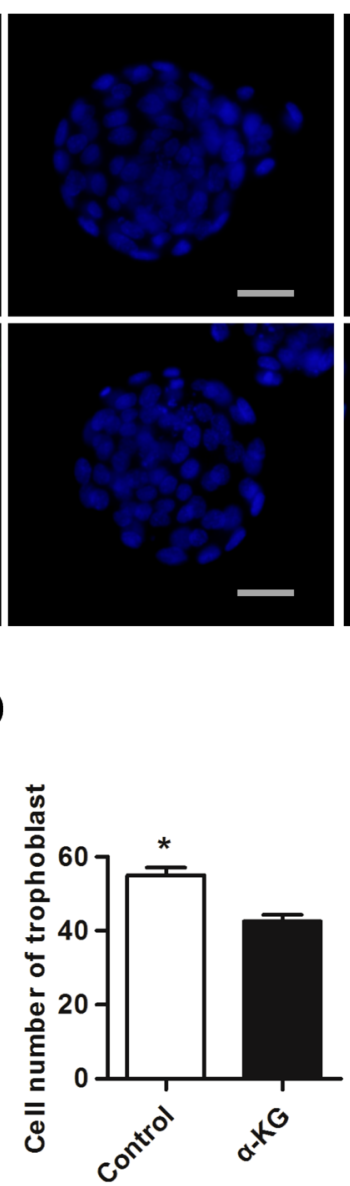

MERGE



E

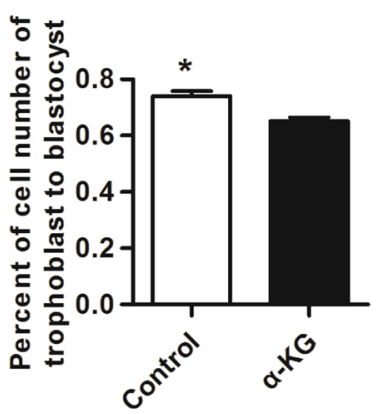

F

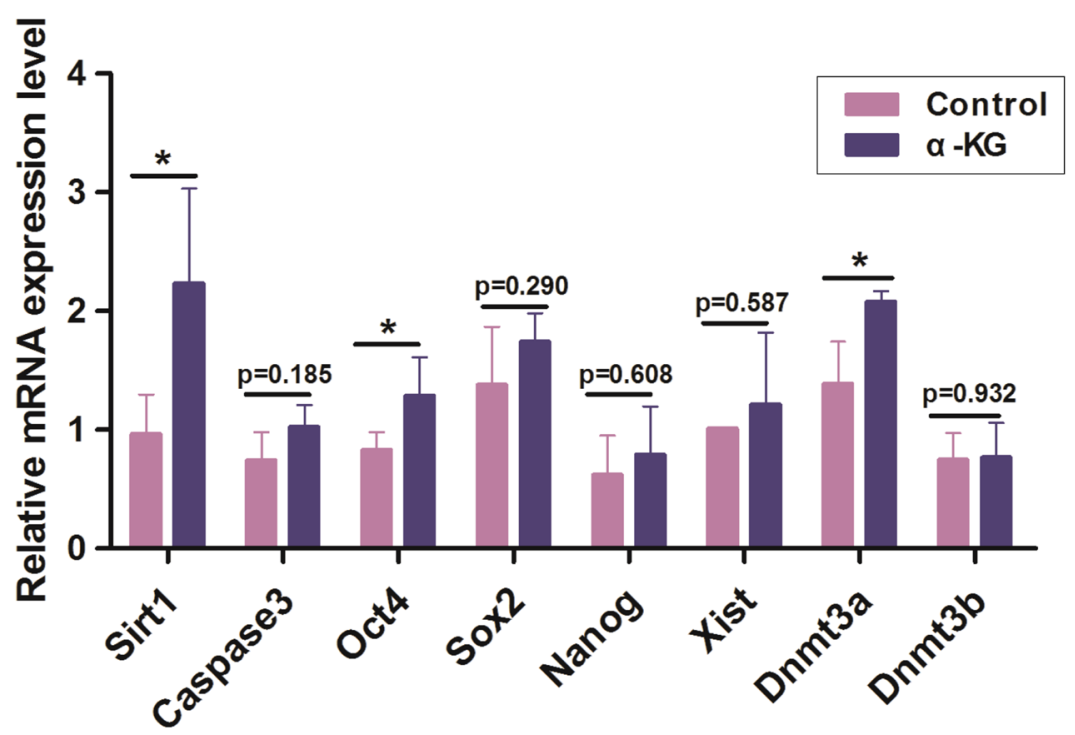

Figure 3 Effects of $\alpha-K G$ on ICM and TE cell number and gene expression levels in blastocysts. (A) Images of the ICM and TE with immunofluorescence staining of OCT4 and CDX2. Red fluorescence: Oct4; green fluorescence: Cdx2; blue fluorescence: DAPI. (B) Average ICM cell number (control, $n=41 ; \alpha-K G, n=55$ ). (C) Average ICM ratio (control, $n=41 ; \alpha-K G, n=55$ ). (D) Average TE cell number (control, $n=41$; $\alpha-K G, n=55$ ). (E) Average TE cell ratio (control, $n=41 ; \alpha-K G, n=55$ ). (F) Effects of $\alpha$-KG on apoptosis-related (Caspase-3), pluripotency-related (Sirt1, Oct4, Sox2, Nanog), X chromosome-related (Xist) and methylation-related (Dnmt3a, Dnmt3b) gene expression. $n=8$ replicates. ${ }^{*} P<0.05$. Scale bars, $20 \mu \mathrm{m}(\mathrm{A})$. 

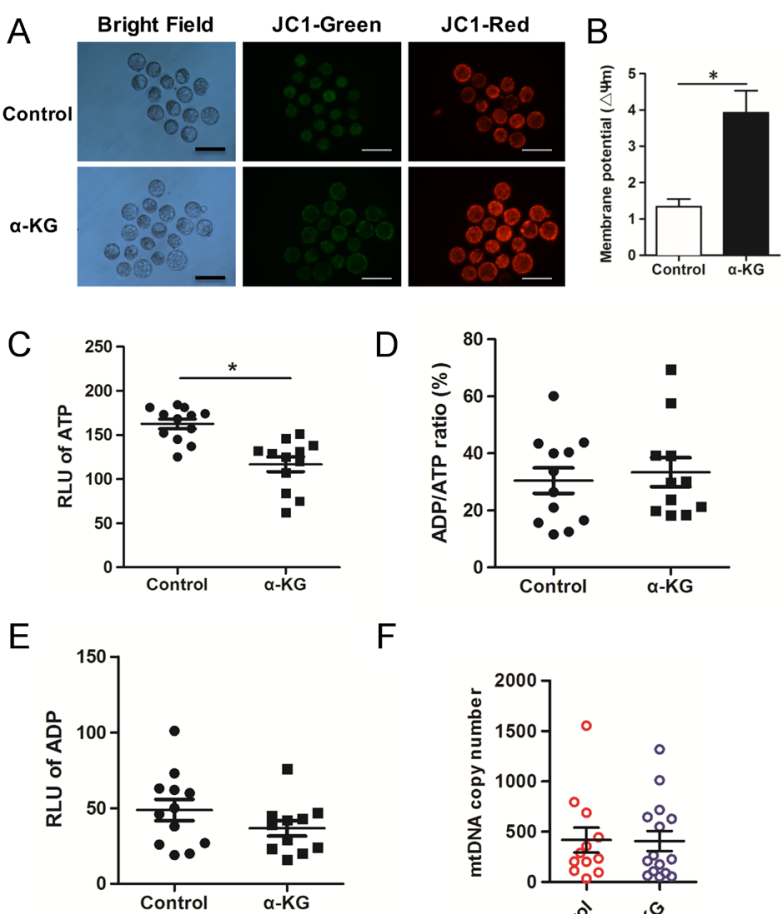

F



Figure 4 Effects of $\alpha-K G$ on the ATP level and mitochondrial copy number in embryos. (A) Images of the mitochondrial membrane potential (MMP) with JC-1 staining. Red fluorescence: high MMP; green fluorescence: low MMP. (B) Statistical analysis of mitochondrial membrane potential (control, $n=13 ; \alpha-K G, n=18$ ); (C and E) Statistical analysis of relative luminescence (RLU) indicating the ATP and ADP level (control, $n=7 ; \alpha-K G, n=7 ; n$ indicates replicates). (D) The ADP/ATP ratio in embryos (control, $n=7 ; \alpha-K G, n=7 ; n$ indicates replicates). (F) Mitochondrial copy number (control, $n=10 ; \alpha-K G$, $n=11 ; n$ indicates replicates). ${ }^{*}(P<0.05)$.

among the groups (Fig. 3F). The apoptosis rates of blastocysts treated with $\alpha-K G$ at $150 \mu \mathrm{M}$ were similar $(7.42 \pm 1.02 \%)$ to those of the controls $(6.26 \pm 0.66 \%)$ (Supplementary Fig. 2).

\section{$\alpha-K G$ alters iron ion-related genes in early embryos based on RNASeq analysis}

To further investigate the molecular mechanism underlying the beneficial effect of $\alpha-K G$ on blastocyst development and implantation, a high-throughput mRNA sequencing analysis was performed. The results showed that approximately 96 genes were upregulated and 40 genes were downregulated by $\alpha-K G(150 \mu \mathrm{M})$ treatment (Fig. 5A). Given that $\alpha-K G$ is an important energy metabolite in the TCA cycle, mitochondriarelated gene expression was a focus. The results indicated that $\alpha-K G(150 \mu \mathrm{M})$ treatment had limited effects on mitochondria-related gene expression levels (Supplementary Table 2). Only one gene (Ucp1) was downregulated and five genes (Gm4952, Gdap1,

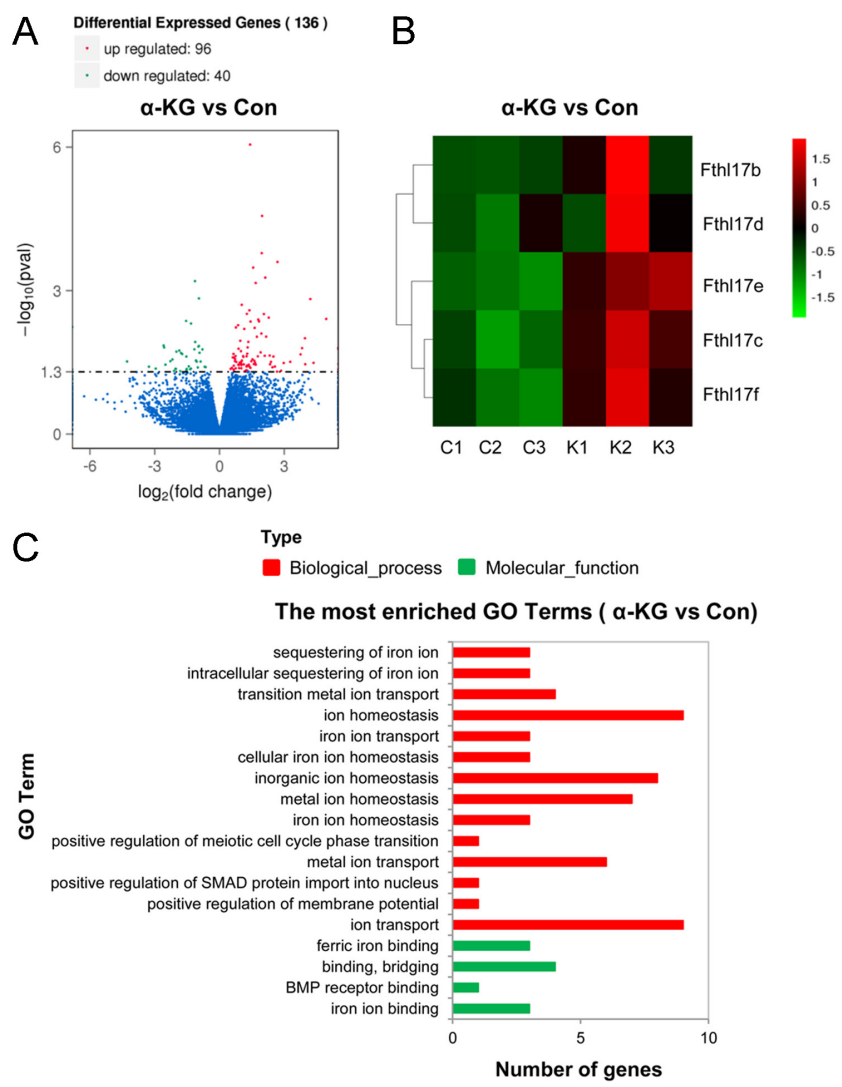

Figure 5 Effects of $\alpha-K G$ on iron-related genes in early embryos analysed via RNA-seq. (A) Volcano map of significantly upregulated and downregulated genes. (B) Heat map of Fthl17 family genes. (C) The most enriched GO terms.

Cox4i2, Arg2 and Slc25a27(UCP4)) were upregulated with a more than two-fold change after $\alpha-K G$ treatment.

Among the upregulated genes, approximately $19.0 \%$ were distributed on the $\mathrm{X}$ chromosome (Supplementary Fig. 1A), namely, Fth/17 family genes (Fth/17C, Fth/17f, and Fth/17, etc.), which are located on the paternal $X$ chromosome in the ICM of the blastocyst (Kobayashi et al. 2010) (Fig. 5B). Interestingly, the Fth/17 family genes composed the most enriched GO terms - iron ion-related terms, including intracellular sequestering of iron ions, ferric iron binding, iron ion transport and transition metal ion transport, among others (Fig. 5C).

\section{$\alpha-K G$ increases the $5 \mathrm{hmC} / 5 \mathrm{mC}$ ratio and TET enzyme activity in embryos}

Compared with in vivo blastocysts, the $5 \mathrm{mC}$ and $5 \mathrm{hmC}$ levels in the in vitro embryos were significantly lower. However, the mean fluorescence intensity (M.F.I) representing $5 \mathrm{hmC}$ in the $\alpha-\mathrm{KG}$ treated embryos $(1814.22 \pm 8.63)$ was significantly higher than that in the in vitro controls $(184.19 \pm 61.13 ; P<0.05)$ and lower than that in the in vivo blastocysts $(4250.78 \pm 738.64$; $P<0.05$ ) (Fig. 6A). Similarly, the M.F.I of $5 \mathrm{mC}$ in the 

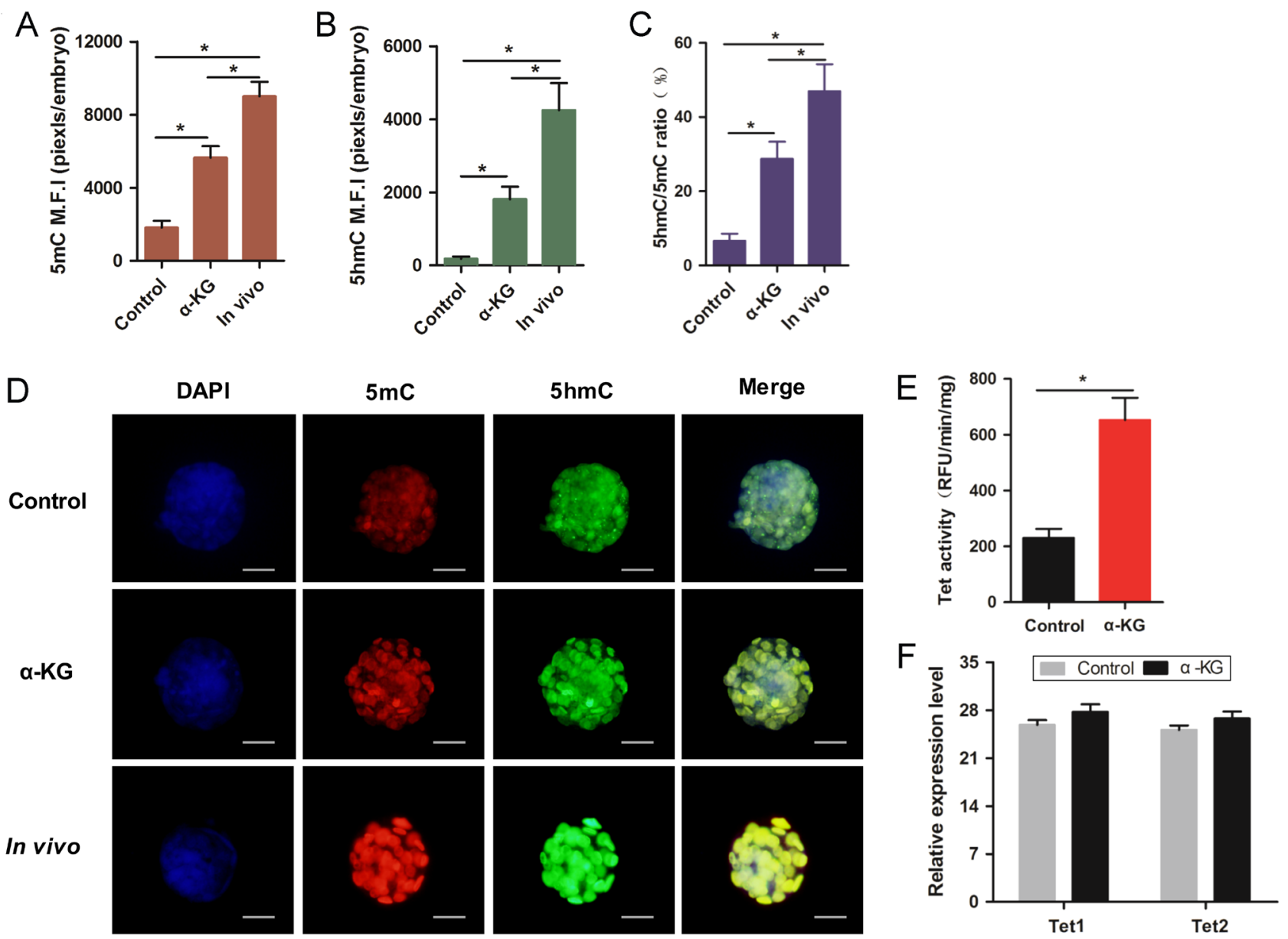

Figure 6 Effects of $\alpha-K G$ on the $5 \mathrm{hmC} / 5 \mathrm{mC}$ ratio and TET enzyme activity in embryos. (A, B and C) Analysis of the mean fluorescence intensity (M.F.I) of $5 \mathrm{mC}, 5 \mathrm{hmC}$ and the $5 \mathrm{hmC} / 5 \mathrm{mC}$ ratio in blastocysts between the in vitro (control and $\alpha-\mathrm{KG}$ ) and in vivo groups (control, $n=20$; $\alpha-\mathrm{KG}$, $n=17$; in vivo, $n=7$ ). (D) Images of $5 \mathrm{hmC}$ and $5 \mathrm{mC}$ with immunofluorescence staining in blastocysts in the in vitro (control and $\alpha-\mathrm{KG}$ ) and in vivo groups. Red, FITC fluorescence-labelled $5 \mathrm{mC}$; green, FITC fluorescence-labelled $5 \mathrm{hmC}$; blue, DAPI-stained chromosomes. (E) The relative enzyme activity of TET. (F) The relative gene expression of Tet1/2 genes determined by qRT-PCR; $n=8$ replicates $* P<0.05$. Scale bars, $20 \mu \mathrm{m}$ (D).

$\alpha-K G$ group (5657.07 \pm 620.87$)$ was significantly higher than that in the in vitro control $(1817.5 \pm 372.03$; $P<0.05)$ and lower than that in the in vivo blastocysts $(9006.00 \pm 795.57 ; P<0.05)$ (Fig. $6 B$ ). The ratio of $5 \mathrm{hmC}$ to $5 \mathrm{mC}$ in the in vivo embryos $(46.92 \pm 6.92 \%)$ was significantly higher than that in the in vitro groups. Under in vitro conditions, the $5 \mathrm{hmC}$ to $5 \mathrm{mC}$ ratio in the $\alpha$-KG group $(28.75 \pm 4.62 \%)$ was significantly higher than that in the control group $(6.61 \pm 1.98 \% ; p<0.05)$ (Fig. 6B). The expression levels of methylation (Dnmt3a/ Dnmt3b) and demethylation (Tet1/Tet2) genes were also detected using qRT-PCR. Dnmt3a showed remarkable upregulation in the $\alpha-K G$ group, but no significant differences were found in Tet 1 , Tet 2 or Dnmt3b between the groups (Figs $3 \mathrm{~F}$ and $6 \mathrm{~F}$ ). We did not compare the expression level of Tet3 in the control and $\alpha-K G$ group because the expression level of Tet3 is extremely low or the gene is not even expressed in the blastocyst stage (Cao et al. 2014, Fan et al. 2015). Similarly, no significant differences were found in the FPKM levels of Tet1, Tet2 and Tet3 between the groups (Supplementary Fig. 1B, C and D). However, the enzyme activity of TET was significantly increased in the $\alpha$-KG group compared with that in the control group according to TET hydroxylase activity quantification (Fig. 6E).

\section{Discussion}

Exposure of murine embryos from the pronuclear to blastocyst stages to $\alpha-K G$ increased the embryo development rate and foetal growth after transfer into recipient female mice. These results indicate that $\alpha$-KG is an important metabolite that promotes embryonic development because $\alpha-K G$ reduces embryo ATP production and activates the TET enzyme, thereby enhancing the ICM and the competence of the blastocyst. The findings were consistent with previous observations that the ICM grade is positively related to birth weight (Licciardi et al. 2015) and the survival rate of transplanted embryos (Otsuki et al. 2016, 


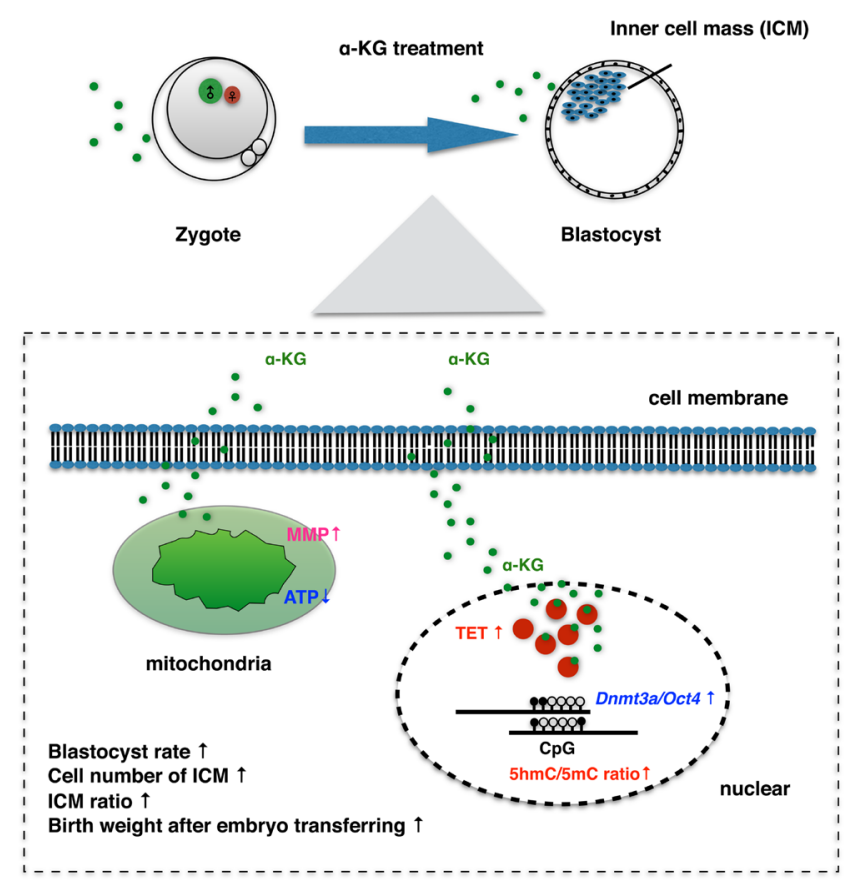

Figure 7 Schematic reprepresentation of $\alpha-K G$ affecting embryo development..

Subira et al. 2016). In humans, blastocysts with larger ICMs are more likely to implant after transfer than those with smaller ICMs (Richter et al. 2001). Machaty et al. (1998) compared the development of early porcine embryos under in vivo and in vitro conditions and found that the ICM cell number and ICM:TE ratio under in vivo conditions were significantly higher than those under in vitro culture conditions.

Surprisingly, $\alpha-K G$ treatment enhanced both the ICM cell number and the ratio while lowering those of the TE in blastocysts. Two potential mechanisms may be involved in these changes. First, dramatic changes in methylation may occur along with cell fate decisions in early embryonic cells (Smith et al. 2014, Legault et al. 2018). A variety of studies have demonstrated that TET1 is required for ICM cell specification in blastocysts (Ito et al. 2010, Williams et al. 2011). $\alpha$-KG is not only a TCA cycle metabolite but is also a factor of dioxygenase. We observed that TET enzyme activity and the $5 \mathrm{hmC}$ to $5 \mathrm{mC}$ ratio were significantly enhanced by $\alpha-K G$. These findings were consistent with those of Carey et al. (2015) who demonstrated that $\alpha-K G$ maintained the pluripotency of mESCs through epigenetic regulation. Second, the increase in the ICM may be related to decreased ATP production in mitochondria because a relatively low $\mathrm{O}_{2}$ level or electron transfer chain (ETC) inhibition maintains pluripotency of ESCs and promotes their development (Ficz et al. 2013). This is the case in our study, and it is consistent with the findings in Caenorhabditis elegans by Chin, RM (Chin et al. 2014), who showed that $\alpha-K G$ could delay ageing by inhibiting
ATPase. Collectively, our data showed that $\alpha-K G$ could affect ICM and TE differentiation by reducing ATP production and regulating epigenetics. In addition, the average litter sizes in the $\alpha-K G$ group were similar to those in the control group, and no significant difference in establishing pregnancy after embryo transfer was observed between the groups (data not shown), which suggests that neither $\alpha-K G$ nor the decreased TE affected embryo implantation.

Another unanticipated finding is the decreased total blastocyst cell number in the $\alpha-K G$ groups. This may be related to iron metabolism. RNAseq data suggested that $\alpha-K G$ could affect iron-related pathways through Fth/17 gene family upregulation. The Fth/17 gene family is closely related to iron ion homeostasis. When $\mathrm{Fe}(\mathrm{III})$ or $\mathrm{Fe}(\mathrm{II})$ was supplied with $\alpha-\mathrm{KG}$ in the embryo culture medium, the total blastocyst cell number was rescued to a normal level, but the blastocyst rate was decreased significantly (data not shown). These findings are consistent with those of Zhao et al. (2016) who found that an iron chelating agent could significantly downregulate cleavage and blastocyst rates in mice. Although the total blastocyst cell number in the $\alpha$-KG group was decreased, it was the same as that observed in embryos in vivo (Rinaudo et al. 2006).

MMP is a critical event in the apoptotic process. In cultured human mesenchymal stem cells (hMSCs), the cell population with a high MMP possessed stronger resistance to apoptotic inducers than a cell population with low MMP (Tsai et al. 2015). In our experiments, the higher $\Delta \psi m$ in the $\alpha-K G$ group led to higher embryo competence.

Oxidative stress is another important factor that affects the quality of embryos. In our study, significantly higher ROS levels were observed in the $\alpha-K G$ group before the morula stage. This is not surprising because in the early stage of the embryo, active and passive DNA demethylation requires Tet3 (Shen et al. 2014, Leese et al. 2015). As an important cofactor of dioxygenases, $\alpha-K G$ can increase the activity of ferrous iron and $\alpha$-KG-dependent dioxygenases ( $\alpha$ KGDs) and enhance ROS production when dioxygen was used as an oxidant to catalyse various reactions via $\mathrm{CH}$ bond activation (Chinopoulos et al. 2013, Wu et al. 2016). The ROS level was not sufficiently high to induce apoptosis based on the low level of TUNEL staining and caspase-3 expression.

Compared with in vivo blastocysts, the $5 \mathrm{mC}$ and $5 \mathrm{hmC}$ levels in the in vitro blastocysts were significantly decreased, which indicates abnormal epigenetic modification. However, $\alpha$-KG-treatment under in vitro conditions significantly increased both the $5 \mathrm{hmC}$ and $5 \mathrm{mC}$ levels in blastocysts compared with those in the in vitro controls, which indicates that $\alpha$-KG potentially preserves some epigenetic properties observed under the in vitro conditions. For example, after $\alpha-K G$ treatment, the FPKM expression levels of Tet1/Tet2/ 
Tet3 and the relative Tet1/Tet2 gene expression levels were controlled to the levels observed under the in vivo conditions, while the enzyme activity of TET was significantly upregulated. The enhanced TET activity, similar to ascorbate (Hore et al. 2016), converts Fe(III) (the most common form of iron in the cell) into Fe(II) to activate the centre of the TET family and enhance the $5 \mathrm{hmC}$ level in the blastocyst. The significantly increased $5 \mathrm{mC}$ level in the $\alpha-K G$ group might be attributed to the remarkable increase in the DNA methylation gene Dnmt3a. The increase in the $5 \mathrm{hmC} / 5 \mathrm{mC}$ ratio in the in vitro embryos induced by $\alpha-K G$ causes epigenetic modifications similar to those observed under in vivo conditions. The increased $5 \mathrm{hmC} / 5 \mathrm{mC}$ ratio may explain the increased ICM and competence of blastocysts. We did not compare the $5 \mathrm{mC} / 5 \mathrm{hmC}$ levels in the ICM and TE cells separately in control and $\alpha-K G$ treated embryos. Since $\alpha-K G$ is a cofactor for the TET enzyme (Carey et al. 2015), we hypothesised that both the ICM and TE may undergo demethylation after $\alpha-K G$ treatment.

Another interesting finding is that significantly differentially expressed genes identified via RNAseq (approximately $19.0 \%$ ) were located on the $X$ chromosome; however, expression of the sex-ratiorelated gene Xist (Tan et al. 2016) was not significantly altered. The birth sex ratio in the control (female $~ 50 \%$, $n=33$ ) and $\alpha$-KG-treated (female $\sim 66 \%, n=21$ ) groups was slightly different but failed to reach statistical significance. Due to the relatively small sample size, no conclusion could be made in this study. $\alpha$-KG may modify certain metabolic pathways and epigenetics, which then impacts X chromosome-imprinted inactivation and leads to an imbalance in the sex ratio. This hypothesis is worthy of follow-up study.

Collectively, we found that $\alpha-K G$ treatment exerted positive effects on embryo development, the ICM ratio and the outcomes of assisted reproduction in mice. The potentially molecular mechanisms are two-fold. First, $\alpha-K G$ regulates mitochondria function to slightly reduce ATP production. The relatively low ATP content was required to maintain the pluripotency of the ICM in the embryo (Fischer et al. 1993, Ezashi et al. 2005, Varum et al. 2009). Second, Fe(II) and $\alpha-K G$ function as cofactors to activate the TET enzyme family to increase DNA demethylation, which also promotes maintenance of the pluripotency of the ICM and competence of the blastocyst (Williams et al. 2011, Carey et al. 2015, Hore et al. 2016). Preservation of the ICM pluripotency is the key factor in the improved embryo quality and development induced by $\alpha-K G$ treatment (Fig. 7).

$\alpha-K G$ is an important energy metabolism intermediate, has no toxic effects and is safe to use. Our findings provide basic knowledge to better understand the potential mechanisms underlying the beneficial effects of $\alpha-K G$ on assisted reproduction in mice and may also be applicable in large animals, including assisted reproduction in humans. Application of these findings in the production of high-quality large animal embryos and ESCs is our future research goal.

\section{Supplementary data}

This is linked to the online version of the paper at https://doi.org/10.1530/REP-19-0018.

\section{Declaration of interest}

The authors declare that there is no conflict of interest that could be perceived as prejudicing the impartiality of the research reported.

\section{Funding}

Guoshi Liu was supported by the National New Biological Breeding Project (2018ZX08008-01B), the National Natural Science Foundation of China (31830091) and Beijing Dairy Industry Innovation Team (BAIC06-2017).

\section{Author contribution statement}

Zhenzhen Zhang, Changjiu He and Guoshi Liu designed the experiments and performed data analysis. Zhenzhen Zhang, Dongying Lv, Tianqi Zhu, Changjiu He, Guangdong Li, HaoWu, Jing Wang and Yukun Song carried out the experiments. Zhenzhen Zhang and Changjiu He wrote the manuscript. Guoshi Liu and Lu Zhang revised the paper and contributed to critical discussions. Guoshi Liu supervised the research group. All the authors read and approved the final manuscript.

\section{References}

Cao SY, Han JY, Wu J, Li QY, Liu SC, Zhang W, Pei YL, Ruan XA, Liu ZH, Wang XM et al. 2014 Specific gene-regulation networks during the pre-implantation development of the pig embryo as revealed by deep sequencing. BMC Genomics 15 4. (https://doi.org/10.1186/1471-216415-4)

Carey BW, Finley LW, Cross JR, Allis CD \& Thompson CB 2015 Intracellular alpha-ketoglutarate maintains the pluripotency of embryonic stem cells. Nature 518 413-416. (https://doi.org/10.1038/nature13981)

Chen J, Guo L, Zhang L, Wu H, Yang J, Liu H, Wang X, Hu X, Gu T, Zhou Z et al. 2013 Vitamin C modulates TET1 function during somatic cell reprogramming. Nature Genetics 45 1504-1509. (https://doi. org/10.1038/ng.2807)

Chin RM, Fu X, Pai MY, Vergnes L, Hwang H, Deng G, Diep S, Lomenick B, Meli VS, Monsalve GC et al. 2014 The metabolite alpha-ketoglutarate extends lifespan by inhibiting ATP synthase and TOR. Nature $\mathbf{5 1 0}$ 397-401. (https://doi.org/10.1038/nature13264)

Chinopoulos C 2013 Which way does the citric acid cycle turn during hypoxia? The critical role of alpha-ketoglutarate dehydrogenase complex. Journal of Neuroscience Research 91 1030-1043. (https://doi. org/10.1002/jnr.23196)

Ezashi T, Das P \& Roberts RM 2005 Low O2 tensions and the prevention of differentiation of hES cells. PNAS 102 4783-4788. (https://doi. org/10.1073/pnas.0501283102)

Fan XY, Zhang XN, Wu XL, Guo HS, Hu YQ, Tang FC \& Huang YY 2015 Single-cell RNA-seq transcriptome analysis of linear and circular RNAs in mouse preimplantation embryos. Genome Biology 16 148. (https:// doi.org/10.1186/s13059-015-0706-1) 
Ficz G, Branco MR, Seisenberger S, Santos F, Krueger F, Hore TA, Marques CJ, Andrews S \& Reik W 2011 Dynamic regulation of 5-hydroxymethylcytosine in mouse ES cells and during differentiation. Nature 473 398-402. (https://doi.org/10.1038/nature10008)

Ficz G, Hore TA, Santos F, Lee HJ, Dean W, Arand J, Krueger F, Oxley D, Paul YL, Walter J et al. 2013 FGF signaling inhibition in ESCs drives rapid genome-wide demethylation to the epigenetic ground state of pluripotency. Cell Stem Cell 13 351-359. (https://doi.org/10.1016/j. stem.2013.06.004)

Fischer B \& Bavister BD 1993 Oxygen tension in the oviduct and uterus of rhesus monkeys, hamsters and rabbits. Journal of Reproduction and Fertility 99 673-679. (https://doi.org/10.1530/jrf.0.0990673)

Hore TA, von Meyenn F, Ravichandran M, Bachman M, Ficz G, Oxley D, Santos F, Balasubramanian S, Jurkowski TP \& Reik W 2016 Retinol and ascorbate drive erasure of epigenetic memory and enhance reprogramming to naive pluripotency by complementary mechanisms. PNAS 113 12202-12207. (https://doi.org/10.1073/pnas.1608679113)

Ito S, D'Alessio AC, Taranova OV, Hong K, Sowers LC \& Zhang Y 2010 Role of Tet proteins in $5 \mathrm{mC}$ to $5 \mathrm{hmC}$ conversion, ES-cell self-renewal and inner cell mass specification. Nature 466 1129-1133. (https://doi. org/10.1038/nature09303)

Ito S, Shen L, Dai Q, Wu SC, Collins LB, Swenberg JA, He C \& Zhang Y 2011 Tet proteins can convert 5-methylcytosine to 5-formylcytosine and 5-carboxylcytosine. Science 333 1300-1303. (https://doi.org/10.1126/ science.1210597)

Jurkowski TP \& Jeltsch A 2011 Burning off DNA methylation: new evidence for oxygen-dependent DNA demethylation. ChemBioChem 12 2543-2545. (https://doi.org/10.1002/cbic.201100549)

Kobayashi S, Fujihara Y, Mise N, Kaseda K, Abe K, Ishino F \& Okabe M 2010 The X-linked imprinted gene family Fthl17 shows predominantly female expression following the two-cell stage in mouse embryos. Nucleic Acids Research 38 3672-3681. (https://doi.org/10.1093/nar/ gkq113)

Leese HJ 2015 History of oocyte and embryo metabolism. Reproduction, Fertility, and Development 27 567-571. (https://doi.org/10.1071/ RD14278)

Legault LM \& Mcgraw S 2018 Dynamics of DNA methylation reprogramming at the single-cell level in early human embryos. Biology of Reproduction 98 610-611. (https://doi.org/10.1093/biolre/ioy026)

Licciardi F, Mccaffrey C, Oh C, Schmidt-Sarosi C \& Mcculloh DH 2015 Birth weight is associated with inner cell mass grade of blastocysts. Fertility and Sterility 103 382.e2-387.e2. (https://doi.org/10.1016/j. fertnstert.2014.10.039)

Machaty Z, Day BN \& Prather RS 1998 Development of early porcine embryos in vitro and in vivo. Biology of Reproduction 59 451-455. (https://doi.org/10.1095/biolreprod59.2.451)

Otsuki J, Iwasaki T, Katada Y, Sato H, Furuhashi K, Tsuji Y, Matsumoto Y \& Shiotani M 2016 Grade and looseness of the inner cell mass may lead to the development of monochorionic diamniotic twins. Fertility and Sterility 106 640-644. (https://doi.org/10.1016/j.fertnstert.2016.05.007)

Ren L, Wang Z, An L, Zhang Z, Tan K, Miao K, Tao L, Cheng L, Zhang Z, Yang $\mathbf{M}$ et al. 2015 Dynamic comparisons of high-resolution expression profiles highlighting mitochondria-related genes between in vivo and in vitro fertilized early mouse embryos. Human Reproduction 30 2892-2911. (https://doi.org/10.1093/humrep/dev228)

Richter KS, Harris DC, Daneshmand ST \& Shapiro BS 2001 Quantitative grading of a human blastocyst: optimal inner cell mass size and shape. Fertility and Sterility 76 1157-1167. (https://doi.org/10.1016/S00150282(01)02870-9)

Rinaudo PF, Giritharan G, Talbi S, Dobson AT \& Schultz RM 2006 Effects of oxygen tension on gene expression in preimplantation mouse embryos. Fertility and Sterility 86 1252.e1-1265.e1. (https://doi.org/10.1016/j. fertnstert.2006.05.017)

Shen L, Inoue A, He J, Liu Y, Lu F \& Zhang Y 2014 Tet3 and DNA replication mediate demethylation of both the maternal and paternal genomes in mouse zygotes. Cell Stem Cell 15 459-471. (https://doi.org/10.1016/j. stem.2014.09.002)

Smith ZD, Chan MM, Humm KC, Karnik R, Mekhoubad S, Regev A, Eggan K \& Meissner A 2014 DNA methylation dynamics of the human preimplantation embryo. Nature 511 611-615. (https://doi.org/10.1038/ nature13581)

Subira J, Craig J, Turner K, Bevan A, Ohuma E, Mcveigh E, Child T \& Fatum M 2016 Grade of the inner cell mass, but not trophectoderm, predicts live birth in fresh blastocyst single transfers. Human Fertility 19 254-261. (https://doi.org/10.1080/14647273.2016.1223357)

Tahiliani M, Koh KP, Shen Y, Pastor WA, Bandukwala H, Brudno Y, Agarwal S, lyer LM, Liu DR, Aravind L et al. 2009 Conversion of 5-methylcytosine to 5-hydroxymethylcytosine in mammalian DNA by MLL partner TET1. Science 324 930-935. (https://doi.org/10.1126/ science.1170116)

Tan K, An L, Miao K, Ren L, Hou Z, Tao L, Zhang Z, Wang X, Xia W, Liu J et al. 2016 Impaired imprinted X chromosome inactivation is responsible for the skewed sex ratio following in vitro fertilization. PNAS 113 3197-3202. (https://doi.org/10.1073/pnas.1523538113)

Tsai AC, Liu Y, Yuan X \& Ma T 2015 Compaction, fusion, and functional activation of three-dimensional human mesenchymal stem cell aggregate. Tissue Engineering: Part A 21 1705-1719. (https://doi.org/10.1089/ten. TEA.2014.0314)

Varum S, Momcilovic O, Castro C, Ben-Yehudah A, Ramalho-Santos J \& Navara CS 2009 Enhancement of human embryonic stem cell pluripotency through inhibition of the mitochondrial respiratory chain. Stem Cell Research 3 142-156. (https://doi.org/10.1016/j. scr.2009.07.002)

von Meyenn F, Iurlaro M, Habibi E, Liu NQ, Salehzadeh-Yazdi A, Santos F, Petrini E, Milagre I, Yu M, Xie Z et al. 2016 Impairment of DNA methylation maintenance is the main cause of global demethylation in naive embryonic stem cells. Molecular Cell 62 983. (https://doi. org/10.1016/j.molcel.2016.06.005)

Wang F, Tian X, Zhang L, Tan D, Reiter RJ \& Liu G 2013 Melatonin promotes the in vitro development of pronuclear embryos and increases the efficiency of blastocyst implantation in murine. Journal of Pineal Research 55 267-274. (https://doi.org/10.1111/jpi.12069)

Williams K, Christensen J, Pedersen MT, Johansen JV, Cloos PA, Rappsilber J \& Helin K 2011 TET1 and hydroxymethylcytosine in transcription and DNA methylation fidelity. Nature 473 343-348. (https://doi.org/10.1038/ nature10066)

Wu RC, Wang Z, Liu MJ, Chen DF \& Yue XS 2004 Beta2-integrins mediate a novel form of chemoresistance in cycloheximide-induced U937 apoptosis. Cellular and Molecular Life Sciences 61 2071-2082. (https:// doi.org/10.1007/s00018-004-4165-1)

Wu LF, Meng S \& Tang GL 2016 Ferrous iron and alpha-ketoglutaratedependent dioxygenases in the biosynthesis of microbial natural products. Biochimica and Biophysica Acta 1864 453-470. (https://doi. org/10.1016/j.bbapap.2016.01.012)

Zernicka-Goetz M, Morris SA \& Bruce AW 2009 Making a firm decision: multifaceted regulation of cell fate in the early mouse embryo. Nature Reviews: Genetics 10 467-477. (https://doi.org/10.1038/nrg2564)

Zhao MH, Liang S, Guo J, Choi JW, Kim NH, Lu WF \& Cui XS 2016 Analysis of ferrous on ten-eleven translocation activity and epigenetic modifications of early mouse embryos by fluorescence microscopy. Microscopy and Microanalysis 22 342-348. (https://doi.org/10.1017/ S1431927616000040)

Received 11 January 2019

First decision 6 March 2019

Revised manuscript received 22 April 2019

Accepted 3 May 2019 